\title{
Eliminating Direction Specificity in Visuomotor Learning
}

\author{
Cong Yin, ${ }^{1,2}$ Yuqing Bi, ${ }^{1,2}$ Cong $Y u,{ }^{1,2,3,4}$ and Kunlin Wei ${ }^{1,2,3}$ \\ ${ }^{1}$ Department of Psychology, ${ }^{2}$ Beijing Key Laboratory of Behavior and Mental Health, ${ }^{3}$ Peking-Tsinghua Center for Life Sciences, and ${ }^{4}$ IDG/McGovern \\ Institute for Brain Research, Peking University, Beijing 100081, China
}

The generalization of learning offers a unique window for investigating the nature of motor learning. Error-based motor learning reportedly cannot generalize to distant directions because the aftereffects are direction specific. This direction specificity is often regarded as evidence that motor adaptation is model-based learning, and is constrained by neuronal tuning characteristics in the primary motor cortices and the cerebellum. However, recent evidence indicates that motor adaptation also involves model-free learning and explicit strategy learning. Using rotation paradigms, here we demonstrate that savings (faster relearning), which is closely related to model-free learning and explicit strategy learning, is also direction specific. However, this new direction specificity can be abolished when the participants receive exposure to the generalization directions via an irrelevant visuomotor gain-learning task. Control evidence indicates that this exposure effect is weakened when direction error signals are absent during gain learning. Therefore, the direction specificity in visuomotor learning is not solely related to model-based learning; it may also result from the impeded expression of model-free learning and explicit strategy learning with untrained directions. Our findings provide new insights into the mechanisms underlying motor learning, and may have important implications for practical applications such as motor rehabilitation.

Key words: learning specificity; motor adaptation; motor generalization; motor learning

\section{Significance Statement}

Motor learning is more useful if it generalizes to untrained scenarios when needed, especially for sports training and motor rehabilitation. However, as a form of motor learning, motor adaptation is typically direction specific. Here we first show that savings with motor adaptation, an index for model-free learning and explicit strategy learning in motor learning, is also direction specific. However, the participants' additional exposure to untrained directions via an irrelevant gain-learning task can enable the complete generalization of learning. Our findings challenge existing models of motor generalization and may have important implications for practical applications.

\section{Introduction}

Learning will not be very meaningful if it is confined to its original learning context. Motor learning generalization examines how learning in one context influences the performance in untrained contexts and offers a unique window for investigating the nature of motor learning (Poggio and Bizzi, 2004; Shadmehr, 2004). Adaptation paradigms have been frequently used to study motor learning generalization in the context of reaching, where arm movements are systematically perturbed by visual distortion

Received July 17, 2015; revised Feb. 17, 2016; accepted Feb. 20, 2016.

Author contributions: C. Yin, C. Yu, and K.W. designed research; C. Yin and Y.B. performed research; C. Yin and K.W. analyzed data; C. Yin, C. Yu, and K.W. wrote the paper.

This study was supported by Natural Science Foundation of China Grants 31371020, 31328010, 61533001, and 31230030; and by the National High Technology Research and Development Program of China (863 Program, Grant 2012AA011602).

The authors declare no competing financial interests.

Correspondence should be addressed to either Cong Yu or Kunlin Wei, Department of Psychology, Peking University, Beijing 100871, China, E-mail: yucong@pku.edu.cn or wei.kunlin@pku.edu.cn.

DOI:10.1523/JNEUROSCI.2712-15.2016

Copyright $\odot 2016$ the authors $\quad 0270-6474 / 16 / 363839-09 \$ 15.00 / 0$
(Pine et al., 1996; Bock et al., 2001) or robotic forces (Thoroughman and Shadmehr, 2000). Typically, learning obtained with adaptation paradigms in one part of the workspace is not fully generalizable to other parts of the workspace when examined with aftereffects (Krakauer et al., 2000; Wigmore et al., 2002; Donchin et al., 2003; Wang and Sainburg, 2004; Krakauer et al., 2006). A widely accepted view is that learning leads to the formation of internal models, a conceptual construct of how the nervous system predicts the sensory consequence of motor commands in the face of perturbations (Shadmehr et al., 2010). The generalization of this learned construct is thought to be constrained by learning-related changes of tuning properties in lower motor cortices, including the primary motor cortex (Thoroughman and Shadmehr, 2000; Paz et al., 2003), the cerebellum (Shadmehr et al., 2010), and the premotor cortex (Wise et al., 1998; Krakauer et al., 2004).

However, recent findings suggest that motor adaptation, which is traditionally attributed to internal models and cerebellum-based learning, consists of model-free learning components (Diedrichsen et al., 2010; Huang et al., 2011; Verstynen and Sabes, 2011; Shmuelof 
A

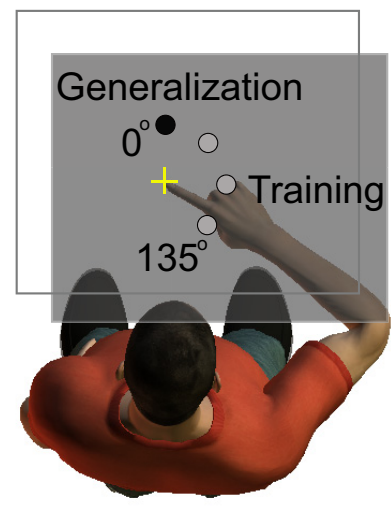

B

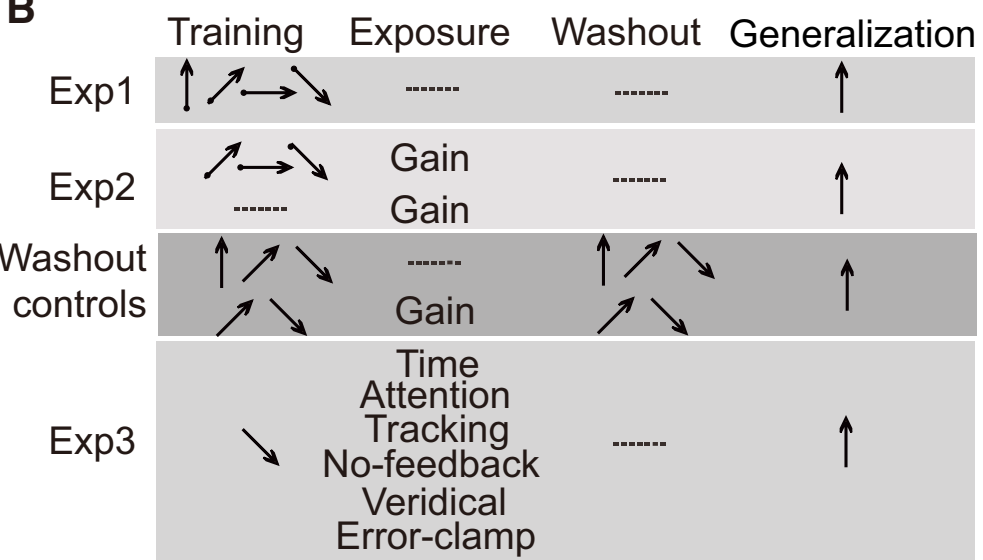

Figure 1. Experimental setup and designs. $\boldsymbol{A}$, An illustration of the experimental setup with reaching targets shown on the screen. $\boldsymbol{B}$, Experimental designs in various experiments. The arrows indicate the learning and generalization directions. The dashed lines indicate that no trial was performed in those specific experimental phases. The exposure tasks were different among groups. Exp, Experiment.

et al., 2012) and explicit strategy-learning components (Taylor et al., 2014; McDougle et al., 2015). Whether these parts of learning are subject to direction specificity is unknown. To investigate this issue, we have to abandon the conventionally used aftereffects and instead use savings (i.e., faster relearning during delayed retest) as the generalization index. In contrast to aftereffect, which reflects the combined effect of all learning components, savings is a behavioral marker for model-free learning and explicit strategy learning (Huang et al., 2011; Haith et al., 2015). Furthermore, savings is a universal metric for all learning systems, including semantic, perceptual, and motor systems (Ebbinghaus, 1913; Liu and Weinshall, 2000; Medina et al., 2001; Kojima et al., 2004; Lebrón et al., 2004; Krakauer et al., 2006). In contrast, aftereffect is exclusively used in motor adaptation paradigms.

Parallel to motor learning, visual perceptual learning is known to be specific to the trained stimulus location or feature dimension (e.g., a specific orientation or direction). Early models often attribute learning specificity to neuronal tuning changes in early visual areas (Karni and Sagi, 1993; Schoups et al., 1995; Teich and Qian, 2003). However, perceptual learning becomes completely transferrable if the participants are additionally exposed to the transfer location or feature dimension via performing an irrelevant task (Xiao et al., 2008; Zhang et al., 2010; Wang et al., 2012; Zhang and Yang, 2014). These results suggest that visual perceptual learning is high-level rule-based learning that occurs beyond the retinotopic and feature-selective early visual cortex. Encouraged by these perceptual learning findings, we hypothesized that if motor learning (savings) is also direction specific, it may be generalizable to distant directions with proper direction exposures. This is highly likely given that motor learning also involves high-level model-free learning and explicit strategy learning.

In this study, we tested this hypothesis by studying the generalization of visuomotor rotation adaptation using savings as a metric of learning. We first found direction-limited generalization of initial rotation learning. However, after exposing the participants to distant generalization directions with visuomotor gain adaptation, a distinct and irrelevant visuomotor learning task (Turner et al., 2003), we demonstrated complete generalization of original learning to distant directions.

\section{Materials and Methods}

\section{Participants}

Two hundred two right-handed participants (81 males; average age, $22.0 \pm 3.5$ years) were recruited ( $n=12$ for each of the 16 experimental groups and $n=10$ for a control group in Experiment 2). All participants were inexperienced, naive to the purpose of the study, and paid for their time. Informed consent, which was approved by the Peking University Institutional Review Board, was obtained from each participant before testing.

\section{Experimental setup}

The participants sat behind a desk and moved their right hand on the desktop. Their vision of the hand and arm was blocked by a semisilvered mirror placed horizontally at the chest level. The movement of the index finger tip was measured at $200 \mathrm{~Hz}$ by a motion capture system (Codamotion). Visual feedback was projected top-down from an LCD projector (display frequency, $75 \mathrm{~Hz}$; model P1270, Acer) mounted $1.45 \mathrm{~m}$ above a horizontally placed back-projection screen. The image was then reflected in the mirror for the participants to view (Fig. 1A). The actual hand was not visible.

The participants were instructed to make straight, center-out shooting movements to a target in successive attempts. Possible targets, shown as white discs with a 4 pixel diameter, were displayed $70 \mathrm{~mm}$ from the starting position. The straight-ahead target was defined as a $0^{\circ}$ target, and the other target directions were labeled as $45^{\circ}, 90^{\circ}$, and $135^{\circ}$ in a clockwise fashion (Fig. 1A). During visuomotor training, the participants experienced a $30^{\circ}$ counterclockwise visuomotor rotation. They needed to move $30^{\circ}$ clockwise to compensate for the imposed rotation.

\section{Procedure}

At the beginning of each trial, the participants rested the right index finger on a $4 \mathrm{~mm}$ plastic disc glued onto the desktop. The disc was also used as an anchor to guide the participants to return the unseen finger to the starting position after each trial. A visual starting position, depicted as a yellow cross (4 pixels per line), was overlaid on the plastic disc. A cursor (a green disc, 4 pixels in diameter) representing the finger-tip position was visible only within $4 \mathrm{~mm}$ around the starting position. Once the finger remained at the starting point for $100 \mathrm{~ms}$, a target appeared, and a computer speaker beeped to signal the participants to move. A beep sound was also used to signal the participants to bring the finger back to the starting position for the next trial once they completed the movement. A low-pitched tone warned the participants when they moved too fast or too slowly (movement time, $<100$ or $>400 \mathrm{~ms}$ ). Before formal data collection, the participants familiarized themselves with the required movement speed. 


\section{Experimental design}

Experiment 1 (generalization without exposure). Each participant was trained at one of the four possible directions (either $0^{\circ}, 45^{\circ}, 90^{\circ}$, or $135^{\circ}$ ) but was tested at the same generalization direction $\left(0^{\circ}\right)$. They were randomly assigned to one of these four training groups. The experiment consisted of the following four consecutive phases: familiarization, baseline, training, and generalization (Fig. $1 B$ ). During the familiarization phase, each participant moved to the training and the generalization targets with veridical and continuous cursor feedback. Each target was visited 10 times, and the order of the targets was randomized. The baseline phase was identical to the familiarization phase except that only the end-point position of the cursor was displayed. This phase was to establish participants' movement baseline for moving toward these targets with end-point feedback. In the training phase, the participants moved to the training target 80 times with visuomotor rotation imposed. The feedback was provided at the end point only. In the generalization phase, the participants moved to the $0^{\circ}$ target 80 times with the same perturbation and end-point feedback as for evaluation of the savings. These four groups were labeled as No-Exposure groups (see below).

Experiment 2 (generalization enabled by visuomotor gain learning). Another three groups of participants (Gain-Exposure groups) were tested for the effect of a secondary gain-learning task on motor generalization. The procedure was the same as that in Experiment 1, except that there was a brief exposure phase between the training and generalization phases. Each group was trained for a single direction at $45^{\circ}, 90^{\circ}$, or $135^{\circ}$. During the exposure phase, the participants moved 20 times to the $0^{\circ}$ generalization target with visuomotor gain perturbation. Gain learning and rotation learning are distinct learning processes, as they are governed by different neural substrates (Turner et al., 2003; Krakauer et al., 2004) and can be obtained independently and concurrently (Yin and Wei, 2014). The gain between the actual movement and its display was set at 0.6 (a veridical gain was 1), while the direction feedback was veridical. Therefore, the participants should move $42 \mathrm{~mm}$ for the visually $70 \mathrm{~mm}$ away target to compensate for the imposed gain. After the exposure phase, all groups were tested again for their generalization at the $0^{\circ}$ direction. To exclude the possibility that the exposure task alone could lead to generalization of rotation learning, a control group skipped the initial training phase, and completed only the exposure and generalization phases.

Washout controls (savings after washout). Experiments 1 and 2 showed that savings was direction specific and that it could fully generalize after the direction exposure (see Results). However, the aftereffect of initial training was not brought to baseline at the training direction. We thus repeated Experiments 1 and 2 but inserted a washout phase after the initial training. The washout phase included 40 reaching trials to the initial training target with veridical end-point feedback and no rotation perturbation. Similar to Experiment 1, three groups of participants were trained for 80 rotation trials at $0^{\circ}, 45^{\circ}$, and $135^{\circ}$, respectively. After washout, they were tested at the generalization direction of $0^{\circ}$. In addition, similar to Experiment 2, another three groups of participants were trained at $0^{\circ}, 45^{\circ}$, and $135^{\circ}$. They then completed a washout phase at the training direction and an exposure phase (i.e., gain learning) at the generalization direction before the generalization test.

Experiment 3 (relevant factors in the exposure task). To investigate the possible influences that could contribute to exposure-enabled generalization, six groups of participants completed different exposure tasks. The procedure was identical to that of Experiment 2 except that the exposure task varied, and only generalization at $135^{\circ}$ was examined.

The first group (the Time group) examined whether the elapsed time was responsible for exposure-enabled generalization. Between the training and generalization phases, the participants sat idle for $1 \mathrm{~min}$, approximately the same duration as in the original exposure task.

The second group (the Attention group) examined whether merely attending to the generalization direction could enable learning generalization. During the exposure phase, the participants performed a luminance discrimination task around the $0^{\circ}$ generalization target. This visual task demanded that the participants direct their attention to the generalization direction. The task followed a single-trial two-alternative forced-choice staircase procedure for measuring discrimination thresh- olds. In each trial, two squares symmetrically flanked the $0^{\circ}$ target (target not shown) with a randomly chosen deviation of $20^{\circ}, 40^{\circ}$, or $60^{\circ}$. The participants were asked to verbally report which square (left or right) was brighter as quickly as possible. This arrangement forced the participants to visually attend to the area surrounding the generalization target. The experimenter pushed the left or right arrow key to record the responses. A classic 3-down-1-up staircase rule was used with a step size of 4 in a 256 grayscale. The reference luminance was at the 128th level of the grayscale. This exposure task lasted for $\sim 60 \mathrm{~s}$, similar to the duration of the visuomotor gain-learning task in Experiment 2.

The third group (the Tracking group) examined whether the exposure of a motor learning task without reaching movements could also enable generalization. The participants were required to track a moving visual target with the hand cursor as accurately as possible. The movement of the target followed a predefined figure-eight trajectory consisting of two identical ellipses whose two semiaxes were 18 and $5.7 \mathrm{~mm}$ long. The long axes of the two ellipses were aligned with the $0^{\circ}$ target direction. This tracking task typically lasted for 120 s. To facilitate learning, the tracking error within a trial was calculated and presented to the participants after each trial. The tracking error was defined as the root mean square error of the tracking trajectory relative to the target trajectory, as follows:

$$
\text { RMSE }=\sqrt{\left(\sum_{i=1}^{n} \Delta x^{2}+\sum_{i=1}^{n} \Delta y^{2}\right) / n},
$$

where $\Delta x$ and $\Delta y$ were the tracking errors in $x$ - and $y$-coordinates in the screen unit, and RMSE was the root mean squared error. Tong and Flanagan (2003) reported no between-task interferences when a tracking task and a reaching task were successively learned with opposite visuomotor rotations. This result suggests that the memory resources for visuomotor rotations are task specific. It also implies that tracking and reaching are different tasks. Therefore, the performance of the Tracking group would indicate whether the exposure effect can be elicited by learning a motor task that is different from the original learning task.

The fourth group (the No-Feedback group) examined whether the exposure of similar reaching actions without visual feedback could enhance the relearning rate. The participants were required to make shooting movements toward the $0^{\circ}$ generalization target without visual or reward feedback. Note that this straight-shooting movement involved identical muscle activation patterns as in the original rotation learning movements.

The fifth group (the Veridical group) examined whether the exposure of a reaching task with veridical feedback could facilitate relearning. The participants were required to make reaching movements toward the $0^{\circ}$ generalization target with veridical visual feedback.

The sixth and last group (the Error-Clamp group) examined the potential effect of learning from small direction errors. During the exposure of gain learning, small direction errors still existed due to the end-point variance of natural reaching movements. In fact, people learn from their own small errors during unperturbed natural movements (van Beers, 2009). Here the directional errors were completely removed by using error-clamp trials where end-point feedback was projected onto the desired $0^{\circ}$ movement direction.

\section{Data analysis}

The direction error of hand reaching was used to quantify the performance in visuomotor rotation learning. The error was the angular difference between the desired direction (i.e., $30^{\circ}$ clockwise from the target direction) and the actual movement direction. The latter was the direction of the vector between the starting position and the movement end point.

The error in the first generalization trial indicated the aftereffect. Though we did not remove the visual feedback as in previous studies, we only provided end-point feedback at the end of the trial. Thus, the directional error was still a valid indication of the participants' feedforward estimate.

To quantify the savings, we first calculated the average errors over trials $2-9$ in both training and generalization phases. The difference in errors between these two phases indicated changes in learning rate, with faster relearning signifying savings (for a similar treatment, see Krakauer 

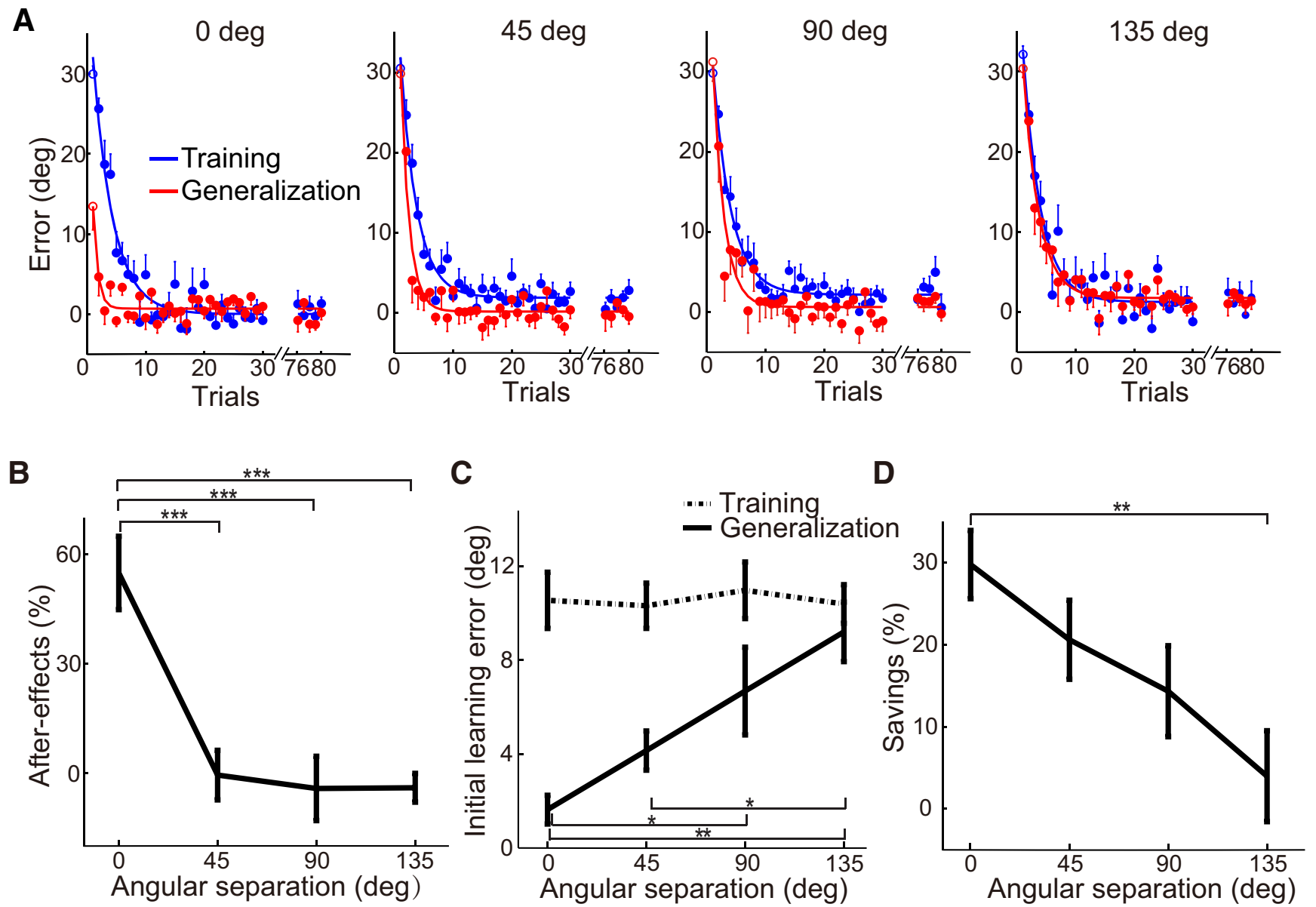

Figure 2. Learning and generalization in Experiment 1. A, Learning over trials during training and generalization, shown separately for the $0^{\circ}, 45^{\circ}, 90^{\circ}$, and $135^{\circ} \mathrm{No}$-Exposure groups. The error bars denote SEMs. Solid lines are fitted exponential learning curves. The first trial (open circle) in the generalization phase is an indicator of the aftereffect. $\boldsymbol{B}$, The aftereffect as a function of the angular separation. C, The initial learning errors in the training (dotted lines) and generalization (solid lines) phases. $\boldsymbol{D}$, The directional generalization quantified by savings. ${ }^{*} p<0.05,{ }^{* *} p<0.01$, *** $p<0.001$.

et al., 2005). We also converted the savings data to percentage data by dividing this difference with the desired directional deviation $\left(30^{\circ}\right)$.

Mixed-design ANOVAs with repeated measures (training and generalization) and fixed measures (groups) were used when multiple groups were compared. Post hoc least significant difference (LSD) tests were performed for simple main effects when there was a significant interaction. The savings of each group was also tested against zero by a onesample $t$ test to detect any successful generalization. The significance level was set at $\alpha=0.05$.

\section{Results}

In the training phase of Experiment 1, four groups of participants learned visuomotor rotation at four different directions $\left(0^{\circ}, 45^{\circ}\right.$, $90^{\circ}$, and $\left.135^{\circ}\right)$, respectively. After initially producing a $30^{\circ}$ error upon the unexpected perturbation, all participants successfully reduced the errors over the ensuing movements and reached a plateau with $\sim 25$ trials (Fig. $2 A$ ). All groups showed similar learning rates as indexed by the average initial learning errors $\left(F_{(3,44)}=0.077, p=0.97\right.$; Fig. $\left.2 C\right)$.

However, the subsequent generalization test, in which all groups were tested at the $0^{\circ}$ direction, exhibited direction specificity. For the first retraining trial, the $0^{\circ}$ group showed higher aftereffects than the other three groups (post hoc LSD test, $p<$ 0.001 ; Fig. $2 B$ ). The aftereffects of the other three groups were not significantly different from zero ( $t$ tests, $p>0.99$ for the $45^{\circ}, 90^{\circ}$, and $135^{\circ}$ groups, respectively). Thus, the learning did not gener- alize to directions $45^{\circ}$ or further away from the training direction in terms of the aftereffects, replicating the direction specificity found in previous studies.

Savings also exhibited direction specificity but with a broader generalization range (Fig. $2 D$ ). In the generalization phase, the initial learning errors were $1.6 \pm 0.6,4.2 \pm 0.8,6.7 \pm 1.9$, and $9.2 \pm 1.3$, respectively, for the $0^{\circ}, 45^{\circ}, 90^{\circ}$, and $135^{\circ}$ directions. Compared with the aftereffects, which showed exponential-like decreases as a function of the angular separation, savings showed almost linear decreases. Some savings was still evident at $45^{\circ}(p<$ $0.001)$ and $90^{\circ}(p<0.05)$, but not at $135^{\circ}(p=0.49)$. A mixeddesign ANOVA with direction as a between-subject factor and phase (training vs generalization) as a within-subject factor confirmed this trend. The ANOVA indicated significant main effects of direction $\left(F_{(3,44)}=3.58, p<0.05\right)$ and phase $\left(F_{(1,44)}=46.21\right.$, $p<0.001)$, as well as their significant interactions $\left(F_{(3,44)}=4.61\right.$, $p<0.01)$. Simple main effects showed no difference between direction groups in the training phase. In contrast, in the generalization phase the learning rate decreased with angular separation: the $0^{\circ}$ group had faster learning than the $90^{\circ}$ and $135^{\circ}$ groups ( $p<0.05$ and 0.005 , respectively), and the $45^{\circ}$ group had faster learning than the $135^{\circ}$ group $(p<0.05)$. The savings were $29.7 \pm 4.2 \%, 20.6 \pm 4.8 \%, 14.3 \pm 5.5 \%$, and $4.0 \pm 5.5 \%$, respectively, for the four directions (Fig. $2 D ; F_{(3,44)}=4.61, p<0.01$, one-way ANOVA). Post hoc pairwise comparison yielded a sig- 

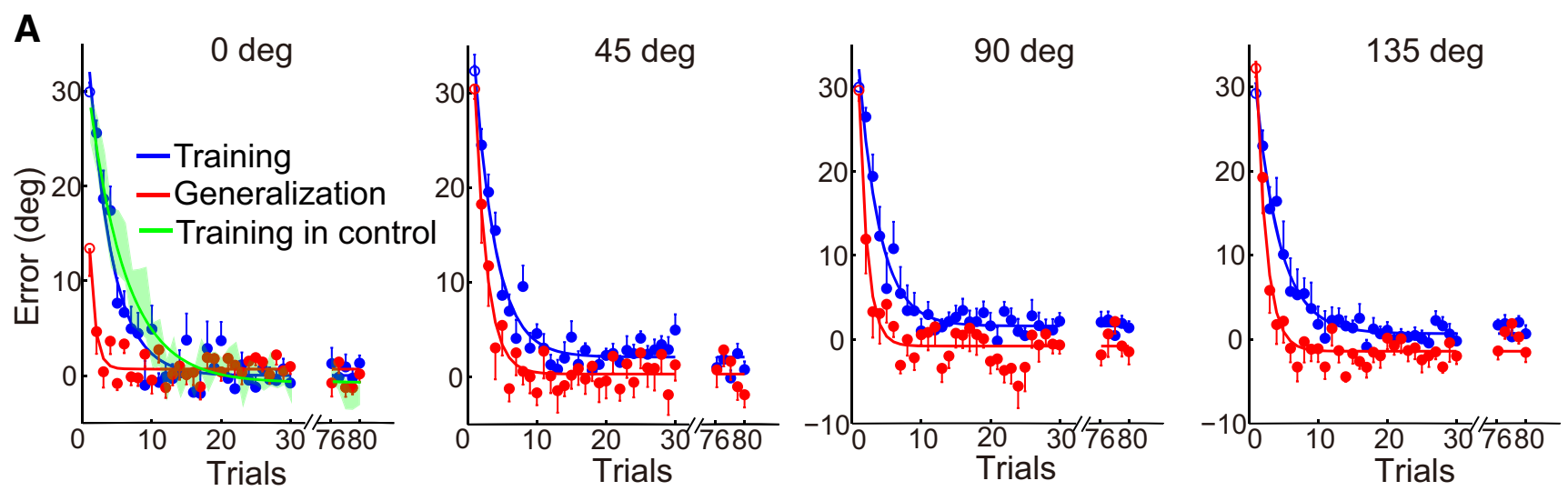

B

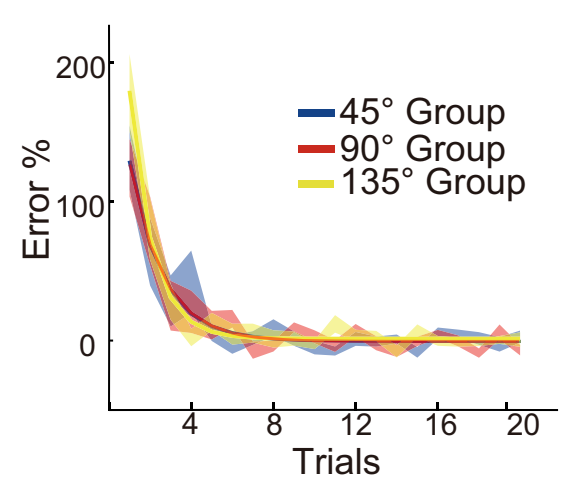

C

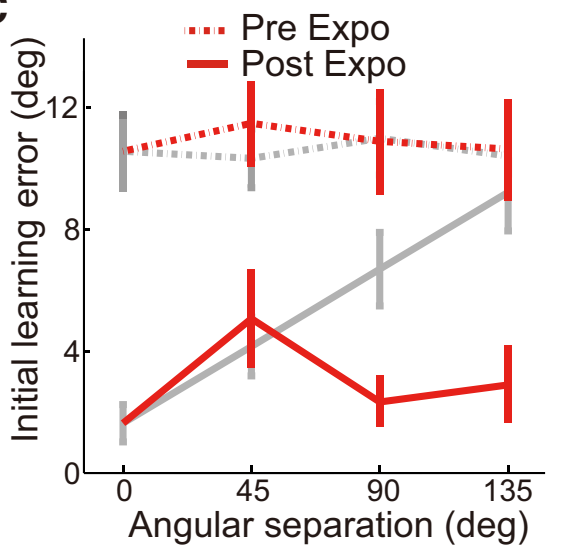

D

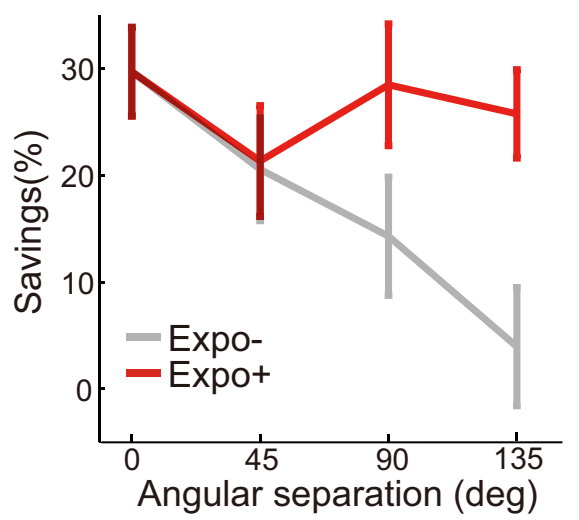

Figure 3. Learning and generalization in Experiment 2 with the exposure of the generation direction via a visuomotor gain-learning task. $\boldsymbol{A}$, Learning during training and generalization at the $0^{\circ}, 45^{\circ}, 90^{\circ}$, and $135^{\circ}$ directions. The error bars denote SEMs. Solid lines are fitted exponential learning curves. The first open circle during the generalization phase indicates the aftereffect. Note the first panel is the $0^{\circ}$ group from Experiment 1 and from the control group who only learned once. $\boldsymbol{B}$, Learning of the visuomotor gain task for the Gain-Exposure groups. Solid lines are fitted exponential learning curves. $\boldsymbol{C}$, Initial learning errors before and after the exposure task (red). The shaded gray lines denote the corresponding initial learning errors in Experiment $1 . \boldsymbol{D}$, The savings is compared between Experiments 1 and 2, which are shown correspondingly by gray and red lines. Expo, Exposure; Pre, before; Post, after.

nificant difference between the $0^{\circ}$ and $135^{\circ}$ groups $(p<0.005)$. Hence, we also demonstrated direction specificity in terms of savings, though this generalization function was broader than those of the aftereffects.

To examine whether learning specificity could be eliminated, three groups of new participants in Experiment 2 received the same visuomotor rotation training at $45^{\circ}, 90^{\circ}$, and $135^{\circ}$ directions, respectively, and subsequent generalization tests at the $0^{\circ}$ direction. The critical addition was the exposure to the generalization direction through visuomotor gain learning before the generalization test. These Gain-Exposure groups achieved similar initial learning as the previous No-Exposure groups in terms of the learning rates $\left(F_{(6,77)}=0.1, p=0.99\right.$; Fig. $\left.3 A\right)$.

All Gain-Exposure groups subsequently learned the visuomotor gain task quickly during the 20-trial exposure phase (Fig. 3B). Importantly, the exposure of distant directions via this secondary task indeed facilitated learning generalization. Though the first generalization trial at $0^{\circ}$ did not exhibit any benefit from previous learning (i.e., no aftereffect), the subsequent learning was substantially faster, indicating a strong savings effect. The learning rates increased, as the initial learning errors amounted to $5.1 \pm$ $1.5^{\circ}, 2.3 \pm 0.7^{\circ}$, and $2.9 \pm 1.2^{\circ}$ in these groups, respectively (Fig. $3 C$ ). A mixed-design ANOVA was performed with group (previous $0^{\circ}$ No-Exposure group vs three Gain-Exposure groups) as a between-subject factor and phase (training vs generalization phases) as a within-subject factor. The results revealed a signifi- cant main effect of phase $\left(F_{(1,44)}=118.1, p<0.001\right)$, but no significant main effect of group $\left(F_{(3,44)}=0.81, p=0.49\right)$ and no significant group $\times$ phase interaction $\left(F_{(3,44)}=0.59, p=0.63\right)$. Therefore, the exposure increased the learning rates in three Gain-Exposure groups to be similar to those with direct training. This complete generalization was further confirmed by directly analyzing the savings. The savings of the three Gain-Exposure groups were $21.4 \pm 5.2 \%, 28.5 \pm 5.7 \%$, and $25.8 \pm 4.1 \%$, respectively (Fig. $3 D$ ), which were not significantly different from the savings of the $0^{\circ}$ No-Exposure group $\left(F_{(3,44)}=0.59, p=0.63\right)$.

To exclude the possibility that the exposure task alone could increase the learning rate, a control group $(n=10)$ skipped the initial training phase and was tested for learning visuomotor rotation right after gain learning. The exposure task apparently did not affect the rotation performance: the learning was not significantly different from the initial learning of the $0^{\circ}$ group in Experiment $1\left(t_{(20)}=-1.03, p=0.31\right.$; replotted and compared in Fig. 3A). Thus, they learned at similar rates as the first-time learners. This was further evidenced by the fact that their learning was significantly slower than the second-time learning for the $0^{\circ}$ No-Exposure group in Experiment $1\left(t_{(20)}=5.01, p<0.001\right)$ and for those who experienced gain exposure in Experiment 2 $\left(t_{(20)}=4.92,5.57\right.$, and 4.86 when compared with the $45^{\circ}, 90^{\circ}$, and $135^{\circ}$ Gain-Exposure groups, respectively; $p$ values $<0.001)$. Therefore, the exposure task alone had no significant impact on learning generalization. It must combine with the 
initial training at a distant direction to enable learning generalization.

The direction specificity and the effect of exposure persisted in the washout control conditions when the aftereffect of initial rotation learning was washed out. Three Non-Exposure groups first showed similar learning rates during initial training, and their relearning rates again showed direction specificity (Fig. 4A, gray). A direction $\times$ phase mixed-design ANOVA showed a significant main effect of phase (training vs generalization, $\left.F_{(1,33)}=13.65, p<0.001\right)$ and a significant interaction effect $\left(F_{(2,33)}=3.70, p<\right.$ $0.05)$. The main effect of direction was not significant $\left(F_{(2,33)}=2.24, p=0.123\right)$. Simple main effects showed no difference among directions in the training phase. In contrast, in the generalization phase, the learning rate decreased with angular separation: the $0^{\circ}$ group had significantly faster learning than the $135^{\circ}$ groups $(p<0.005)$, but the $45^{\circ}$ group was not significantly different from the $0^{\circ}$ and $135^{\circ}$ groups $(p=0.067$ and 0.215$)$. The savings were $25.6 \pm 6.2 \%$, $12.7 \pm 7.6 \%$, and $1.6 \pm 4.5 \%$, respectively, for the $0^{\circ}, 45^{\circ}$, and $135^{\circ}$ directions (Fig. $4 B$; one-way ANOVA, $F_{(2,33)}=3.70, p<$ $0.05)$. Post hoc pairwise comparison yielded a significant difference between the $0^{\circ}$ and $135^{\circ}$ groups $(p<0.01)$. Hence, we demonstrated direction specificity of savings after washout.

Importantly, in another three groups with exposure to the generalization direction, the direction specificity was again removed. A mixed-design ANOVA was performed with group (previous $0^{\circ}$ No-Exposure group vs two Gain-Exposure groups) as a between-subject factor and phase (training vs generalization phases) as a within-subject factor. The results showed no significant main effect of group $\left(F_{(2,33)}=0.81, p=0.49\right)$, a significant main effect of phase $\left(F_{(1,33)}=62.49, p<0.001\right)$, and no significant interaction effect $\left(F_{(2,33)}=0.35, p=0.71\right)$. Therefore, the exposure enabled full generalization after washout (Fig. $4 A)$. This complete generalization was further confirmed by directly analyzing the savings (Fig. 4B). The savings of two Gain-Exposure groups at $45^{\circ}$ and $135^{\circ}$ were $20.3 \pm 3.7 \%$ and $20.9 \pm 4.5 \%$, respectively, which were not significantly different from the savings of the $0^{\circ}$ No-Exposure group $\left(F_{(2,44)}=0.35, p=0.71\right)$. Therefore, washout of initial learning did not change the results observed in Experiments 1 and 2: savings exhibited direction specificity that could be removed by exposure to the generalization direction via a secondary gain-learning task.

To understand why direction exposure enabled generalization, six groups of participants each completed a different exposure task (the Attention, Time, Tracking, No-Feedback, Veridical, and ErrorClamp groups; see Materials and Methods). The separation was $135^{\circ}$ where the enhancement effect was most apparent in the previous Gain-Exposure task (Fig. 5). Proper visual attention to the generalization direction by the Attention group was indicated by luminance discrimination learning (Fig. 5A). Similarly, active engagement of the tracking task by the Tracking group was indicated by tracking learning (Fig. 5B).

All of these groups started off with similar initial learning rates (Fig. 5C,D). Their initial learning errors were not significantly different from those of the previous $135^{\circ}$ No-Exposure group and $135^{\circ}$ Gain-Exposure group $\left(F_{(7,88)}=0.15, p=0.99\right)$. These groups then performed different exposure tasks. In the general- ization phase, their learning was again tested against the $135^{\circ}$ No-Exposure group and $135^{\circ}$ Gain-Exposure group, and a significant difference was found $\left(F_{(7,88)}=2.45, p<0.05\right)$. Post hoc LSD tests revealed that these groups learned significantly more slowly than the original $135^{\circ}$ Gain-Exposure group since the only difference was between the $135^{\circ}$ Gain-Exposure group and other groups $(p=0.007,0.001,0.001,0.006,0.028,0.028$, and 0.056 , respectively, when compared with the $135^{\circ}$ No-Exposure, Attention, Time, Tracking, No-Feedback, Veridical and Error-Clamp groups). Only the Gain-Exposure group and the Error-Clamp group had significant savings $\left(t_{(11)}=6.22\right.$ and 2.48 , and $p<$ 0.001 and $p<0.05$, respectively), and the Veridical group had marginally significant savings $\left(t_{(11)}=2.09, p=0.06\right)$. The savings of the Time, Attention, Tracking, and No-Feedback groups were not significantly different from zero $\left(t_{(11)}=-0.09,-0.02,1.3\right.$, and $0.89 ; p=0.93,0.99,0.22$, and 0.39 , respectively). We did not run a two-way ANOVA, given that these groups were independently sampled and the number of groups was large, thus the error variance was inflated.

\section{Discussion}

Our study demonstrates that savings in visuomotor rotation learning, like the aftereffect, is direction specific. This new direction specificity persisted after a washout session that brought initial learning back to the baseline before relearning. Interestingly, direction exposure with a visuomotor gain-learning task enables savings to fully generalize to distant directions. Control conditions showed that gain learning with no directional error feedback, and reaching with veridical feedback but without gain learning, lead to partial generalization. Thus, exposure to learning and direction errors may be necessary for full generalization of original rotation learning.

Motor generalization is traditionally indicated by the aftereffects, which exhibit direction specificity. This behavioral generalization function has been linked to neuronal tuning properties in primary motor cortices such as M1 and the cerebellum (Donchin et al., 2003; Paz et al., 2003; Shadmehr, 2004). More recent reweighting models that combine the population-coding model and the state space model propose that learning changes the connections between fixed-tuning population neurons with error signals (Poggio and Bizzi, 2004; Tanaka et al., 2009). In this latter framework, motor learning and generalization are manifestations of these weight changes, which are incrementally altered 
A
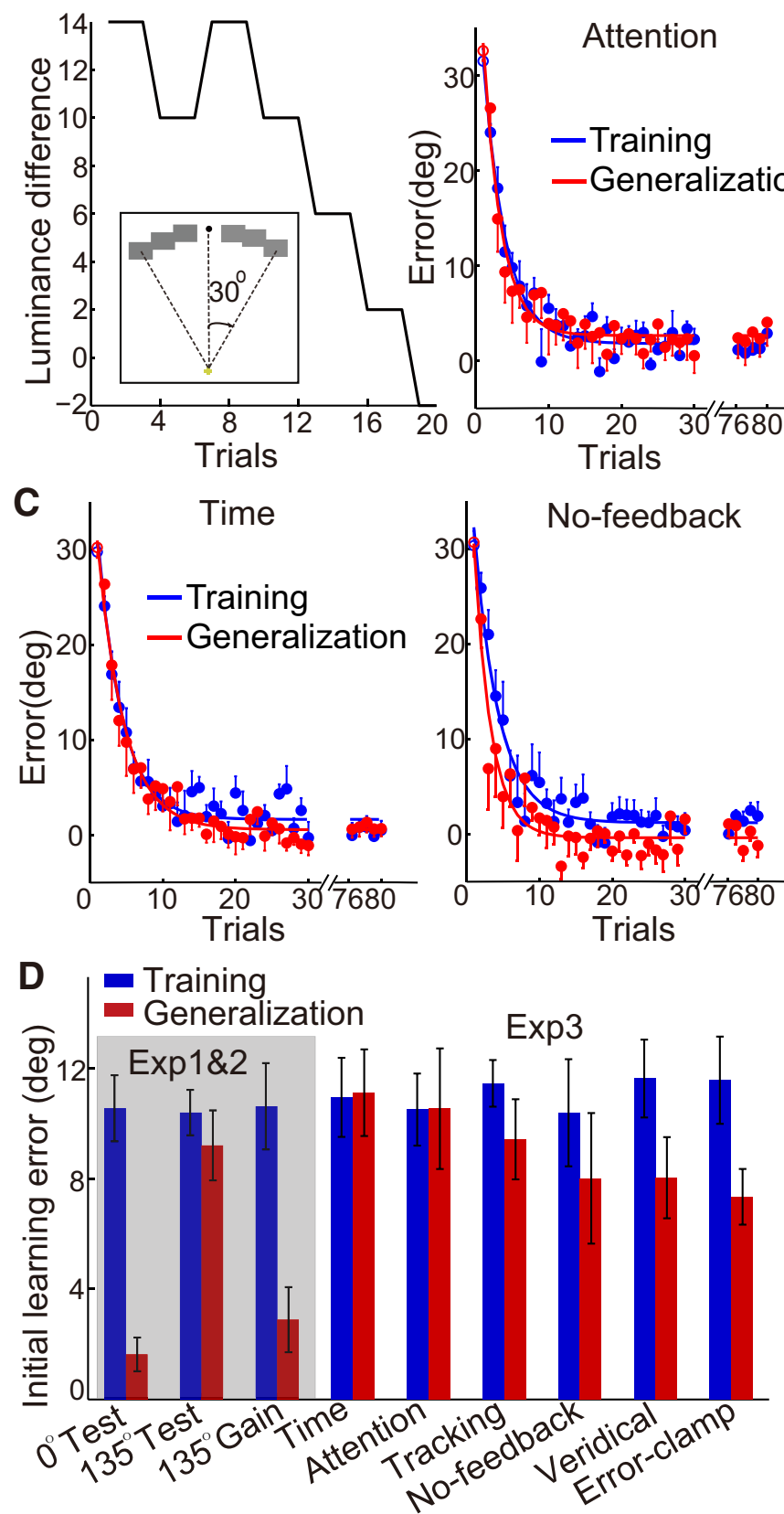

B
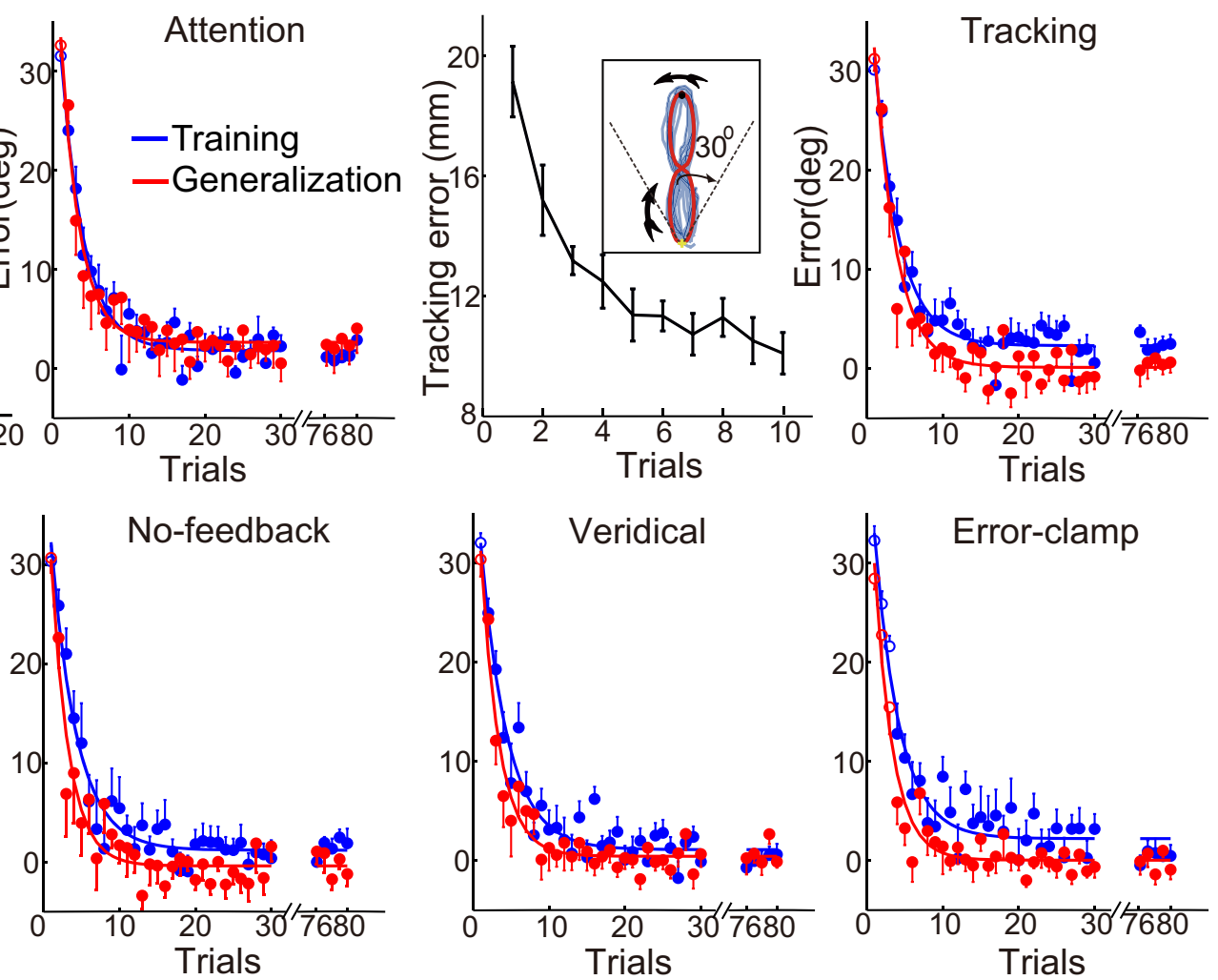

E

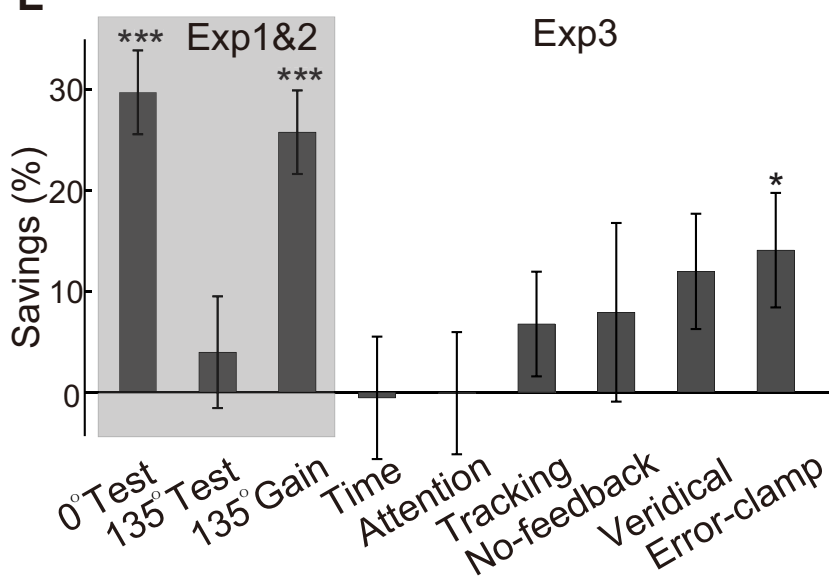

Figure 5. Results from Experiment 3 where different exposure tasks were used. $A$, Luminance discrimination as the exposure task in the Attention group. Left, A typical participant's luminance discrimination learning curve. The inset illustrates possible positions of the stimuli, which symmetrically flanked the to-be-generalized target. Right, The training and generalization functions. $\boldsymbol{B}$, Visuomotor tracking task as the exposure task in the Tracking group. Left, A typical participant's tracking learning curve. The inset illustrates the path of the moving target (red, not shown to the participant), overlaid by exemplary tracking trajectories. Right, The training and generalization functions. C, Rotation learning for other exposure task groups. $\boldsymbol{D}$, Initial learning errors before and after exposure. Results from Experiments 1 and 2 are shown in shades. E, Savings for different exposure task groups. Error bars denote the SEM. Significant differences from zero are marked. ${ }^{* * *} p<$ $0.001,{ }^{*} p<0.05$. Exp, Experiment.

by error signals. Thus, these generalization models are based on the concept of internal models and the measurement of aftereffects.

In contrast, savings reflects distinct learning components of motor adaptation, including reinforcement learning (Huang et al., 2011) and explicit strategy learning (Haith et al., 2015). Reinforcement learning, a form of model-free learning, is an operant association between the adapted movement and the successful error reduction (Diedrichsen et al., 2010; Huang et al., 2011; Verstynen and Sabes, 2011; Shmuelof et al., 2012). From a different perspective, motor adaptation is also divided into an explicit component, which is closely related to cognitive strategies, and an implicit component (Anguera et al., 2010; Fernandez-Ruiz et al., 2011; Taylor et al., 2014; McDougle et al., 2015).

The direction specificity of savings may reflect fundamental constraints on cognitive strategy and operant reinforcement acquired during motor adaptation. In this light, the nervous system has difficulty in applying a newly acquired cognitive strategy (e.g., aiming to a rotated direction) or a reinforced action (adaptive 
rotation for direction errors) to distant directions. We cannot distinguish between these two possibilities as they may be conceptually equivalent, both referring to an aiming strategy in the face of directional perturbations. Requiring the participants to explicitly report the aiming direction may help distinguish the explicit strategy from other learning components (Taylor et al., 2014). Limited generalization is not unexpected, though. In an uncertain environment, any task is associated with a large solution space of possible actions; and the nervous system shall not completely generalize the acquired control policy to any novel situation. The constraints for generalizing motor learning, especially those tied to reinforcement learning and cognitive strategy, demand further investigations.

The intriguing finding is that direction exposure with a seemingly irrelevant learning task can lead to full generalization. Similar muscle-activation patterns in the exposure tasks cannot account for this effect since both of those performed by the Tracking and the No-Feedback groups involve upper arm movements similar to those in the Gain-Exposure groups. In fact, the No-Feedback group used identical ballistic movements and muscle activations as the Gain-Exposure groups. However, neither the Tracking nor the No-Feedback group showed any generalization effect. Both gain learning with visual error clamp and simple reaching with veridical feedback induced partial generalization. Admittedly, reaching with veridical feedback also involves learning where people correct for their own motor errors (van Beers, 2009). We thus postulate that both learning and experiencing original error signals facilitate the nervous system to generalize the acquired strategy to new directions. The gain-learning task involves learning of a novel visuomotor map, a learning feature that also exists in the original rotation learning. Thus, direction exposure via gain learning may induce meta-learning, so that the nervous system infers that novel visuomotor mapping is applicable to a distant direction and consequently expedites the relearning of rotation. This possibility is consistent with recent findings that causal inference is an inherent component of motor learning (Wei and Körding, 2009).

In addition, experiencing directional errors, albeit small ones, can facilitate generalization. Savings has been related to heightened sensitivity to related movement errors (Herzfeld et al., 2014). Our exposure task may help the nervous system quickly recognize rotation errors and adapt to them more quickly during relearning. We also noticed that the first generalization trial, with or without exposure, showed no sign of aftereffect at distant directions. From this perspective, the nervous system may need at least one trial to probe the new direction before applying previously acquired learning (Klassen et al., 2005; Krakauer et al., 2005).

It is unlikely that the direction specificity of savings is caused by use-dependent learning, which is also a form of model-free learning. Use-dependent learning is a movement bias toward the adapted movement repeated at the learning asymptote. Thus, it can potentially lead to direction specificity if its influence decreases at distant directions. However, we found that after exposure to reaching movements $30^{\circ}$ anti-clockwise from the desired solution (Gain-Exposure groups), savings can fully generalize. If use-dependent plasticity is at work, this exposure should only reduce generalization since the initial rotation training is $30^{\circ}$ clockwise. Thus, consistent with a previous report that usedependent plasticity is not sufficient for savings (Huang et al., 2011), our results suggest that the direction specificity of savings and its elimination are not related to use-dependent learning.

The exposure-induced motor generalization is in line with the transfer of visual perceptual learning enabled by a training-plus- exposure paradigm (Xiao et al., 2008; Zhang et al., 2010; Wang et al., 2012, 2016; Zhang and Yang, 2014). Perceptual learning becomes completely transferrable to untrained conditions after additional exposure of these conditions via an irrelevant task. These findings challenge perceptual learning theories that rely on learning-induced plasticity in early visual cortices (Karni and Sagi, 1993; Schoups et al., 1995; Teich and Qian, 2003). Similarly, low-level neural circuits in the primary motor cortex and the cerebellum have been assumed to constrain the generalization of motor learning across directions (Thoroughman and Shadmehr, 2000; Donchin et al., 2003; Paz et al., 2003; Shadmehr, 2004). Based on our findings, we suggest that motor generalization should also engage an extensive range of brain regions, including the striatum and the prefrontal and parietal cortices, which are closely related to reinforcement learning and cognitive learning (Tanaka et al., 2009; Anguera et al., 2010; Wächter et al., 2010).

Our findings are also in line with previous reports about the top-down influence on the generalization of motor learning. For instance, prior motor experience (Krakauer et al., 2006; Wei et al., 2014) and the participants' familiarity with the learning materials (Yan et al., 2013) can enhance the generalization, which was indexed by aftereffects. Note that these studies indicate that the enhancement in generalization is typically associated with longterm exposure of the same or similar learning tasks. For instance, daily experience with a computer mouse leads to enhanced generalization of visuomotor gain learning (Wei et al., 2014). The mouse use and visuomotor gain learning share similar visuomotor transformation, in which the gain between the movement and its visual representation is modified from the veridical one-toone mapping. Our current study goes further to show that learning can completely generalize to distant directions with a brief exposure via an irrelevant learning task.

\section{References}

Anguera JA, Reuter-Lorenz PA, Willingham DT, Seidler RD (2010) Contributions of spatial working memory to visuomotor learning. J Cogn Neurosci 22:1917-1930. CrossRef Medline

Bock O, Schneider S, Bloomberg J (2001) Conditions for interference versus facilitation during sequential sensorimotor adaptation. Exp Brain Res 138:359-365. CrossRef Medline

Diedrichsen J, White O, Newman D, Lally N (2010) Use-dependent and error-based learning of motor behaviors. J Neurosci 30:5159-5166. CrossRef Medline

Donchin O, Francis JT, Shadmehr R (2003) Quantifying generalization from trial-by-trial behavior of adaptive systems that learn with basis functions: theory and experiments in human motor control. J Neurosci 23: 9032-9045. Medline

Ebbinghaus H (1913) Memory: a contribution to experimental psychology. New York: Teachers College, Columbia University.

Fernandez-Ruiz J, Wong W, Armstrong IT, Flanagan JR (2011) Relation between reaction time and reach errors during visuomotor adaptation. Behav Brain Res 219:8-14. CrossRef Medline

Haith AM, Huberdeau DM, Krakauer JW (2015) The influence of movement preparation time on the expression of visuomotor learning and savings. J Neurosci 35:5109-5117. CrossRef Medline

Herzfeld DJ, Vaswani PA, Marko MK, Shadmehr R (2014) A memory of errors in sensorimotor learning. Science 345:1349-1353. CrossRef Medline

Huang VS, Haith A, Mazzoni P, Krakauer JW (2011) Rethinking motor learning and savings in adaptation paradigms: model-free memory for successful actions combines with internal models. Neuron 70:787-801. CrossRef Medline

Karni A, Sagi D (1993) The time course of learning a visual skill. Nature 365:250-252. CrossRef Medline

Klassen J, Tong C, Flanagan JR (2005) Learning and recall of incremental kinematic and dynamic sensorimotor transformations. Exp Brain Res 164:250-259. CrossRef Medline 
Kojima Y, Iwamoto Y, Yoshida K (2004) Memory of learning facilitates saccadic adaptation in the monkey. J Neurosci 24:7531-7539. CrossRef Medline

Krakauer JW, Pine ZM, Ghilardi MF, Ghez C (2000) Learning of visuomotor transformations for vectorial planning of reaching trajectories. J Neurosci 20:8916-8924. Medline

Krakauer JW, Ghilardi MF, Mentis M, Barnes A, Veytsman M, Eidelberg D, Ghez C (2004) Differential cortical and subcortical activations in learning rotations and gains for reaching: a PET study. J Neurophysiol 91: 924-933. CrossRef Medline

Krakauer JW, Ghez C, Ghilardi MF (2005) Adaptation to visuomotor transformations: consolidation, interference, and forgetting. J Neurosci 25: 473-478. CrossRef Medline

Krakauer JW, Mazzoni P, Ghazizadeh A, Ravindran R, Shadmehr R (2006) Generalization of motor learning depends on the history of prior action. PLoS Biol 4:e316. CrossRef Medline

Lebrón K, Milad MR, Quirk GJ (2004) Delayed recall of fear extinction in rats with lesions of ventral medial prefrontal cortex. Learn Mem 11: 544-548. CrossRef Medline

Liu Z, Weinshall D (2000) Mechanisms of generalization in perceptual learning. Vision Res 40:97-109. CrossRef Medline

McDougle SD, Bond KM, Taylor JA (2015) Explicit and implicit processes constitute the fast and slow processes of sensorimotor learning. J Neurosci 35:9568-9579. CrossRef Medline

Medina JF, Garcia KS, Mauk MD (2001) A mechanism for savings in the cerebellum. J Neurosci 21:4081-4089. Medline

Paz R, Boraud T, Natan C, Bergman H, Vaadia E (2003) Preparatory activity in motor cortex reflects learning of local visuomotor skills. Nat Neurosci 6:882-890. CrossRef Medline

Pine ZM, Krakauer JW, Gordon J, Ghez C (1996) Learning of scaling factors and reference axes for reaching movements. Neuroreport 7:2357-2361. CrossRef Medline

Poggio T, Bizzi E (2004) Generalization in vision and motor control. Nature 431:768-774. CrossRef Medline

Schoups AA, Vogels R, Orban GA (1995) Human perceptual learning in identifying the oblique orientation: retinotopy, orientation specificity and monocularity. J Physiol 483:797-810. CrossRef Medline

Shadmehr R (2004) Generalization as a behavioral window to the neural mechanisms of learning internal models. Hum Mov Sci 23:543-568. CrossRef Medline

Shadmehr R, Smith MA, Krakauer JW (2010) Error correction, sensory prediction, and adaptation in motor control. Annu Rev Neurosci 33:89-108. CrossRef Medline

Shmuelof L, Huang VS, Haith AM, Delnicki RJ, Mazzoni P, Krakauer JW (2012) Overcoming motor "forgetting" through reinforcement of learned actions. J Neurosci 32:14617-14621. CrossRef Medline

Tanaka H, Sejnowski TJ, Krakauer JW (2009) Adaptation to visuomotor rotation through interaction between posterior parietal and motor cortical areas. J Neurophysiol 102:2921-2932. CrossRef Medline

Taylor JA, Krakauer JW, Ivry RB (2014) Explicit and implicit contributions to learning in a sensorimotor adaptation task. J Neurosci 34:3023-3032. CrossRef Medline

Teich AF, Qian N (2003) Learning and adaptation in a recurrent model of
V1 orientation selectivity. J Neurophysiol 89:2086-2100. CrossRef Medline

Thoroughman KA, Shadmehr R (2000) Learning of action through adaptive combination of motor primitives. Nature 407:742-747. CrossRef Medline

Tong C, Flanagan JR (2003) Task-specific internal models for kinematic transformations. J Neurophysiol 90:578-585. CrossRef Medline

Turner RS, Desmurget M, Grethe J, Crutcher MD, Grafton ST (2003) Motor subcircuits mediating the control of movement extent and speed. J Neurophysiol 90:3958-3966. CrossRef Medline

van Beers RJ (2009) Motor learning is optimally tuned to the properties of motor noise. Neuron 63:406-417. CrossRef Medline

Verstynen T, Sabes PN (2011) How each movement changes the next: an experimental and theoretical study of fast adaptive priors in reaching. J Neurosci 31:10050-10059. CrossRef Medline

Wächter T, Röhrich S, Frank A, Molina-Luna K, Pekanovic A, Hertler B, Schubring-Giese M, Luft AR (2010) Motor skill learning depends on protein synthesis in the dorsal striatum after training. Exp Brain Res 200:319-323. CrossRef Medline

Wang J, Sainburg RL (2004) Limitations in interlimb transfer of visuomotor rotations. Exp Brain Res 155:1-8. CrossRef Medline

Wang R, Zhang JY, Klein SA, Levi DM, Yu C (2012) Task relevancy and demand modulate double-training enabled transfer of perceptual learning. Vision Res 61:33-38. CrossRef Medline

Wang R, Wang J, Zhang JY, Xie XY, Yang YX, Luo SH, Yu C, Li W (2016) Perceptual learning at a conceptual level. J Neurosci 36:2238-2246. CrossRef Medline

Wei K, Körding K (2009) Relevance of error: what drives motor adaptation? J Neurophysiol 101:655-664. CrossRef Medline

Wei K, Yan X, Kong G, Yin C, Zhang F, Wang Q, Kording KP (2014) Computer use changes generalization of movement learning. Curr Biol 24: 82-85. CrossRef Medline

Wigmore V, Tong C, Flanagan JR (2002) Visuomotor rotations of varying size and direction compete for a single internal model in a motor working memory. J Exp Psychol Hum Percept Perform 28:447-457. CrossRef Medline

Wise SP, Moody SL, Blomstrom KJ, Mitz AR (1998) Changes in motor cortical activity during visuomotor adaptation. Exp Brain Res 121:285-299. CrossRef Medline

Xiao LQ, Zhang JY, Wang R, Klein SA, Levi DM, Yu C (2008) Complete transfer of perceptual learning across retinal locations enabled by double training. Curr Biol 18:1922-1926. CrossRef Medline

Yan X, Wang Q, Lu Z, Stevenson IH, Körding K, Wei K (2013) Generalization of unconstrained reaching with hand-weight changes. J Neurophysiol 109:137-146. CrossRef Medline

Yin C, Wei K (2014) Interference from mere thinking: mental rehearsal temporarily disrupts recall of motor memory. J Neurophysiol 112: 594-602. CrossRef Medline

Zhang JY, Yang YX (2014) Perceptual learning of motion direction discrimination transfers to an opposite direction with TPE training. Vision Res 99:93-98. CrossRef Medline

Zhang JY, Zhang GL, Xiao LQ, Klein SA, Levi DM, Yu C (2010) Rule-based learning explains visual perceptual learning and its specificity and transfer. J Neurosci 30:12323-12328. CrossRef Medline 